\title{
AICAR reduces the collagen-stimulated secretion of PDGF-AB and release of soluble CD40 ligand from human platelets: Suppression of HSP27 phosphorylation via p44/p42 MAP kinase
}

\author{
MASANORI TSUJIMOTO $^{1,2}$, HARUHIKO TOKUDA ${ }^{2,3}$, GEN KUROYANAGI $^{2,4}$, NAOHIRO YAMAMOTO ${ }^{2,4}$, \\ SHINGO KAINUMA ${ }^{2,4}$, RIE MATSUSHIMA-NISHIWAKI ${ }^{2}$, TAKASHI ONUMA ${ }^{2,5}$, YUKO IIDA ${ }^{2,5}$, \\ AKIKO KOJIMA $^{2,5,6}$, SHIGENOBU SAWADA ${ }^{1}$, TOMOAKI DOI ${ }^{7}$, YUKIKO ENOMOTO $^{1}$, \\ KUMIKO TANABE ${ }^{5}$, SHIGERU AKAMATSU ${ }^{6}$, HIROKI IIDA $^{5}$, SHINJI OGURA ${ }^{7}$, \\ TAKANOBU OTSUKA ${ }^{4}$, OSAMU KOZAWA ${ }^{2}$ and TORU IWAMA ${ }^{1}$
}

\author{
Departments of ${ }^{1}$ Neurosugery and ${ }^{2}$ Pharmacology, Gifu University Graduate School of Medicine, Gifu 501-1194; \\ ${ }^{3}$ Department of Clinical Laboratory, National Center for Geriatrics and Gerontology, Obu, Aichi 474-8511; \\ ${ }^{4}$ Department of Orthopedic Surgery, Nagoya City University Graduate School of Medical Sciences, Nagoya, Aichi 467-8603; \\ ${ }^{5}$ Department of Anesthesiology and Pain Medicine, Gifu University Graduate School of Medicine, Gifu 501-1194; \\ ${ }^{6}$ Department of Anesthesiology and Critical Care Medicine, Matsunami General Hospital, Gifu 501-6062; \\ ${ }^{7}$ Department of Emergency and Disaster Medicine, Gifu University Graduate School of Medicine, Gifu 501-1194, Japan
}

Received March 19, 2015; Accepted April 22, 2016

DOI: 10.3892/etm.2016.3435

\begin{abstract}
We have previously reported that collagen-induced phosphorylation of heat shock protein (HSP) 27 via p44/p42 mitogen-activated protein (MAP) kinase in human platelets is sufficient to induce the secretion of platelet-derived growth factor (PDGF)-AB and the release of soluble cluster of differentiation 40 ligand (sCD40L). Adenosine monophosphate-activated protein kinase (AMPK), which is known to regulate energy homeostasis, has a crucial role as an energy sensor in various eukaryotic cells. The present study investigated the effects of 5-aminoimidazole-4-carbox amide-1- $\beta$-d-ribofuranosyl 5'-monophosphate (AICAR), which is an activator of AMPK, on the collagen-induced activation of human platelets. It was demonstrated that AICAR dose-dependently reduced collagen-stimulated platelet aggregation up to $1.0 \mu \mathrm{M}$. Analysis of the size of platelet aggregates demonstrated that AICAR decreased the ratio of large aggregates $(50-70 \mu \mathrm{m})$, whereas the ratio of small aggregates (9-25 $\mu \mathrm{m})$ was increased by AICAR administration. AICAR markedly attenuated the phosphorylation levels of p44/p42 MAP kinase and HSP27, which are induced by collagen.
\end{abstract}

Correspondence to: Dr Haruhiko Tokuda, Department of Clinical Laboratory, National Center for Geriatrics and Gerontology, 7-430 Morioka, Obu, Aichi 474-8511, Japan

E-mail: tokuda@ncgg.go.jp

Key words: adenosine monophosphate-activated protein kinase, collagen, platelet-derived growth factor, soluble cluster of differentiation 40 ligand, platelets
Furthermore, AICAR significantly decreased the secretion of PDGF-AB and the collagen-induced release of SCD40L. These results indicated that AICAR-activated AMPK may reduce the secretion of $\mathrm{PDGF}-\mathrm{AB}$ and the collagen-induced release of $\mathrm{SCD} 40 \mathrm{~L}$ by inhibiting HSP27 phosphorylation via p44/p42 MAP kinase in human platelets.

\section{Introduction}

Platelets have an important role in primary hemostasis, thrombus formation and the repair of vascular injury (1). Platelets are activated by a diverse range of stimulators, leading to alterations in shape, adhesion, aggregation, and subsequent thrombus formation. Collagen is well-documented as a primary stimulator of human platelets $(2,3)$. Platelets interact with collagen in subendothelium at the damaged site of the vessel wall. Therefore, once subendothelium is exposed, platelets rapidly adhere to the exposed subendothelial collagen, which is characterized by the interaction of glycoprotein (GP) Ib/IX/V and von Willebrand factor (1), resulting in aggregation and hemostasis. GPVI and integrin $\alpha 2 \beta 1$ are the predominant collagen receptors located on the plasma membrane of platelets $(2,3)$. GPVI forms a complex with the Fc receptor $\gamma$-chain $(4,5)$. Activated GPVI induces the activation of various intracellular molecules, including phospholipase $C \gamma 2$ and tyrosine kinase Syk $(6,7)$, resulting in the upregulation of integrin activity (8) and the enhancement of granule secretion (2). Platelet-derived growth factor (PDGF)-AB, which is stored in $\alpha$-granules of human platelets and is known to exert potent proliferative effects on a variety of cells, is released from activated platelets and has a pivotal role in atherosclerosis via the proliferation of connective tissue, including vascular smooth muscle cells (1). 
Expression of heat shock proteins (HSPs) is induced in response to various biological stresses, including heat, endotoxins and reactive oxygen species (9). HSPs have been classified into seven subtypes, including HSPA (HSP70), HSPB (low-molecular-weight HSPs) and HSPC (HSP90) (10). It is generally recognized that HSPBs, such as HSP27 and $\alpha \mathrm{B}$-crystallin, possess chaperoning functions as well as HSPA (HSP70) and HSPC (HSP90) (10). Furthermore, it has been demonstrated that the functions of HSP27 are modulated by post-translational modifications, such as phosphorylation $(11,12)$. Human HSP27 is phosphorylated at three serine residues: Ser-15, Ser-78 and Ser-82. Although HSP27 is presented in an aggregated form under unstimulated conditions, it is rapidly dissociated following stimulation-responsive phosphorylation, and it has been demonstrated that this dissociation is necessary for substrate binding and chaperone function (13). HSP27 reportedly increases cell viability under various unfavorable conditions, including heat and oxidative stress $(14,15)$. The phosphorylation of HSP27 in platelets is known to be catalyzed by members of the mitogen-activated protein (MAP) kinase superfamily (16). Furthermore, regarding HSP27 phosphorylation in human platelets, we have previously demonstrated that the collagen-induced phosphorylation of HSP27 via p44/p42 MAP kinase is sufficient for the secretion of PDGF-AB and the release of soluble soluble cluster of differentiation 40 ligand (sCD40L) (17).

Adenosine monophosphate (AMP)-activated protein kinase (AMPK) has a critical role as a regulator of energy homeostasis (18). AMPK is activated under low energy states, including physical exercise, hypoxia and ischemia, which lead to a decrease in the cellular ATP/AMP ratio. It has been demonstrated that AMPK is involved in various physiological signaling pathways associated with the metabolism of glucose, fat and protein, and various processes, such as cell proliferation, apoptosis and aging (19). Previous studies have reported that AMPK is activated by the inhibition of fatty acid synthase, resulting in the cytotoxicity observed in ovarian cancer cells $(20,21)$. Therefore, AMPK is considered as a potential therapeutic target for the treatment of diabetes mellitus (DM), cancer and obesity. Regarding the antiplatelet effect of AMPK (22), it has previously been reported that platelet aggregation is suppressed by 5 -aminoimidazole-4-carboxamide-1- $\beta$-d-ribofuranosyl 5 '-monophosphate (AICAR), which is an activator of AMPK (23). However, the exact mechanism underlying the effects of AMPK on human platelet functions are yet to be clarified.

In the present study, the effects of AICAR on collagen-induced platelet activation were examined in human platelets.

\section{Materials and methods}

Reagents and materials. Collagen was purchased from Takeda Austria GmbH (Linz, Austria). AICAR was purchased from Sigma-Aldrich (St.Louis, MO, USA). PDGF-AB ELISA kit and sCD40L ELISA kit were purchased from R\&D Systems, Inc., (Minneapolis, MN, USA). Phosphorylated (p)-p44/p42 MAP kinase rabbit anti-human polyclonal antibody (cat. no. 9101), p44/p42 MAP kinase rabbit anti-human polyclonal antibody (cat. no. 9102), p-HSP27 (Ser-15) rabbit anti-human polyclonal antibody (cat. no. 2404), p-HSP27 (Ser-78) rabbit anti-human polyclonal antibody (cat. no. 2405) and p-HSP27 (Ser-82) rabbit anti-human polyclonal antibody (cat. no. 2401) were purchased from Cell Signaling Technology, Inc., (Beverly, MA, USA). HSP27 goat polyclonal antibodies (cat. no. sc-1049) and GAPDH rabbit polyclonal antibodies (cat. no. sc-25778) were purchased from Santa Cruz Biotechnology, Inc., (Santa Cruz, CA, USA). Enhanced chemiluminescence (ECL) reagent was purchased from GE Healthcare (Chalfont, UK).

Preparation of platelets. Human blood $(10 \mathrm{ml})$ was donated by healthy volunteers (mean age, 39.2 \pm 9.0 years; mean \pm standard deviation) and supplemented with $3.8 \%$ sodium citrate (1:10). Platelet-rich plasma (PRP) was obtained from the blood samples by centrifugation at $155 \mathrm{x}$ g for $12 \mathrm{~min}$ at room temperature. Platelet-poor plasma (PPP) was prepared from residual blood by centrifugation at $1,400 \mathrm{x}$ g for $5 \mathrm{~min}$ at room temperature. Written informed consent was obtained from all participants signed an informed consent following a detailed explanation of the study. The protocol of the present study was approved by the Committee of Ethics at Gifu University Graduate School of Medicine (Gifu, Japan).

Measurement of platelet aggregation induced by collagen. Platelet aggregation analysis of citrated PRP was performed using an aggregometer (PA-200; Kowa Co. Ltd., Tokyo, Japan), which determines the size of platelet aggregates based on a particle counting laser light scattering system at $37^{\circ} \mathrm{C}$ with a stirring speed of $800 \mathrm{rpm}$. Aggregate sizes were determined as follows: Small, 9-25 $\mu \mathrm{m}$; medium, 25-50 $\mu \mathrm{m}$; and large, $50-70 \mu \mathrm{m}$. Platelets were preincubated for $1 \mathrm{~min}$ prior to platelet aggregation monitoring for $4 \mathrm{~min}$. Percentage transmittance of the isolated platelets was recorded as $0 \%$, and the appropriate PPP blank was recorded as $100 \%$. When indicated, PRP was pretreated with AICAR for $15 \mathrm{~min}$.

Measurement of PDGF-AB and SCD4OL levels. Following stimulation by collagen, platelet aggregation was terminated by the addition of $10 \mathrm{mM}$ ice-cold EDTA solution and the mixture was subsequently centrifuged at $10,000 \mathrm{x}$ g for $2 \mathrm{~min}$ at $4^{\circ} \mathrm{C}$. In order to measure PDGF-AB and sCD40L levels, the supernatant was isolated and stored at $-20^{\circ} \mathrm{C}$. PDGF-AB and sCD40L levels were determined using respective ELISA kits, according to the manufacturer's protocol.

Western blot analysis. As described, following stimulation by collagen, platelet aggregation was terminated and the mixture was subsequently centrifuged at $10,000 \mathrm{xg}$ for $2 \mathrm{~min}$ at $4^{\circ} \mathrm{C}$. For western blot analysis, the pellet was washed twice with phosphate-buffered saline, lysed and immediately boiled in a lysis buffer containing $62.5 \mathrm{mM}$ Tris/Cl (pH 6.8), $2 \%$ sodium dodecyl sulfate (SDS), $50 \mathrm{mM}$ dithiothreitol and $10 \%$ glycerol. Western blot analysis was performed as previously described (24). Briefly, proteins were separated by SDS-polyacrylamide gel electrophoresis PAGE according to the Laemmli method (25) with $10 \%$ or $12 \%$ polyacrylamide gel, and were subsequently transferred onto a polyvinylidine fluoride (PVDF) membranes. Following blocking with 5\% fat-free dry milk in Tris-buffered saline with Tween 20 [TBS-T; $20 \mathrm{mM}$ Tris (pH 7.6), $137 \mathrm{mM} \mathrm{NaCl}, 0.1 \%$ Tween 20] for $2 \mathrm{~h}$ 
A
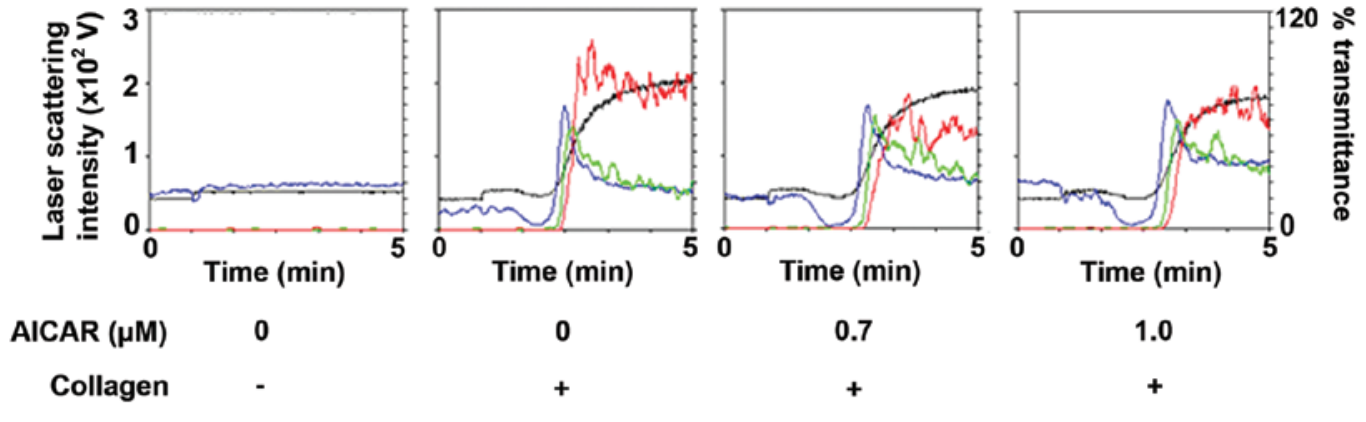

0.7

1.0

\begin{tabular}{rrrrr} 
B & \multicolumn{1}{c}{ Ratio (\%) } & 29 & 42 & 47 \\
\hline small & 100 & 23 & 28 & 25 \\
medium & 0 & 48 & 30 & 28 \\
\hline large & 0 & 100 & 100 & 100
\end{tabular}

Figure 1. Effect of AICAR on collagen-induced platelet aggregation. (A) Following treatment with various doses of AICAR for 15 min and stimulation by $1.0 \mu \mathrm{g} / \mathrm{ml}$ collagen or vehicle for $5 \mathrm{~min}$, platelet aggregation was detected by an aggregometer with laser light scattering. Black line, percentage transmittance of each sample (isolated platelets were recorded as $0 \%$ and platelet-free plasma as $100 \%)$; blue line, small aggregates $(9-25 \mu \mathrm{m})$; green line, medium aggregates $(25-50 \mu \mathrm{m})$; and red line, large aggregates $(50-70 \mu \mathrm{m})$. (B) Distributions (\%) of aggregated particle size were estimated by the area under the curve of each particle size. Representative results obtained from four healthy donors are presented. AICAR, 5 -aminoimidazole-4-carboxamide-1- $\beta$-d-ribofuranosyl 5'-monophosphate.

at room temperature, the membranes were incubated with the primary antibodies p-p44/p42 MAP kinase, p44/p42 MAP kinase, p-HSP27 (Ser-15, Ser-78 and Ser-82) and GAPDH at a dilution of $1: 1,000$ in $5 \%$ milk in TBS-T overnight at $4{ }^{\circ} \mathrm{C}$. Following washing, the membranes were incubated with anti-rabbit (cat. no. 7074S; Cell Signaling Technology, Inc.) or anti-goat (cat. no. sc-2020; Santa Cruz Biotechnology, Inc.) IgG secondary antibodies at a dilution of 1:1,000 in 5\% milk in TBS-T for $1 \mathrm{~h}$ at room temperature. Using an enhanced chemiluminescence western blotting detection system, peroxidase activity on PVDF membranes was visualized on X-ray film, according to the manufacturer's protocol. The densitometry of bands were analyzed by Image $\mathbf{J}$ software program. The quantitative data of each bands were measured as the counts of pixels.

Statistical analysis. Data were analyzed using a paired t-test. $\mathrm{P}<0.05$ was considered to indicate a statistically significant difference. All data are presented as the mean \pm standard error of the mean. All statistical analyses were performed using PASW Statistics software, version 18 (SPSS Japan, Tokyo, Japan).

\section{Results}

Effects of AICAR on collagen induced platelet aggregation. The effect of AICAR on collagen-stimulated platelet aggregation was determined in human platelets. AICAR administration markedly reduced the platelet aggregation induced by collagen in a dose-dependent manner up to $1.0 \mu \mathrm{M}$ (Fig. 1A). Furthermore, analysis of the size of platelet aggregates demonstrated that treatment with $1 \mu \mathrm{m}$ AICAR decreased the ratio of large aggregates $(50-70 \mu \mathrm{m})$ from 48 to $28 \%$; whereas the ratio of small aggregates $(9-25 \mu \mathrm{m})$ increased from 29 to $47 \%$ (Fig. 1B), as compared with the control. These results suggest that AICAR inhibits collagen-stimulated human platelet aggregation.

Effects of AICAR on the phosphorylation of p44/p42 MAP kinase and HSP27 induced by collagen in human platelets. We have previously reported that the collagen-induced phosphorylation of HSP27 is positively regulated by the activation of p44/p42 MAP kinase in human platelets (17). In order to clarify whether AICAR administration affects the collagen-induced activation of p44/p42 MAPK in human platelets, the effect of AICAR on the collagen-induced phosphorylation of p44/p42 MAP kinase was examined using western blot analysis. AICAR administration significantly suppressed collagen-stimulated p44/p42 MAP kinase phosphorylation in a dose-dependent manner up to $1.0 \mu \mathrm{M}$, as compared with the control $(\mathrm{P}<0.05$; Fig. 2).

Subsequently, the effect of AICAR on the collagen-induced phosphorylation of HSP27 was assessed in human platelets. AICAR markedly reduced the collagen-induced phosphorylation of HSP27 at ser-15, ser-78 and ser-82 in a dose-dependent manner up to $1.0 \mu \mathrm{M}$, and significant suppression of phosphorylation was induced by AICAR on Ser-78 and Ser-82 (both $\mathrm{P}<0.05$; Fig. 3). The maximum suppressive effects of AICAR $(1.0 \mu \mathrm{M})$ on the collagen-stimulated phosphorylation of HSP27 at ser-78 and ser- 82 induced a $\sim 40 \%$ and $90 \%$ reduction in the collagen-effect, respectively. These findings suggest that AMPK activating AICAR limits the phosphorylation of HSP27 via the suppression of p44/p42 MAP kinase activity in collagen stimulated human platelets.

Effects of AICAR on the collagen-induced PDGF-AB secretion or $S C D 40 L$ release from human platelets. We have previously demonstrated that the collagen-induced phosphorylation of HSP27 via p44/p42 MAP kinase in human platelets is sufficient for PDGF-AB secretion and SCD40L release (17). 
$\mathbf{A}$

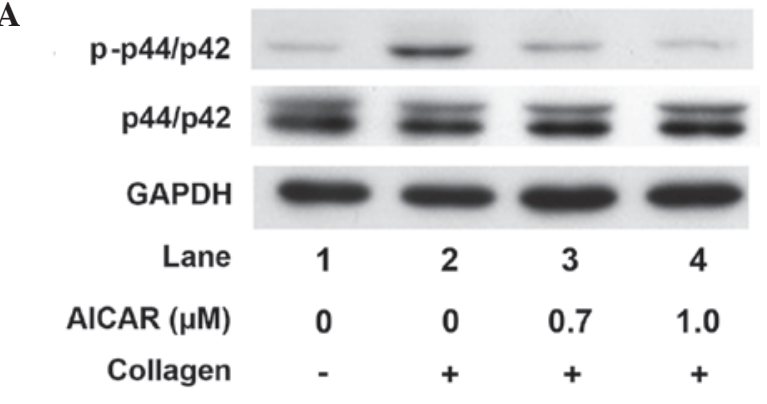

B

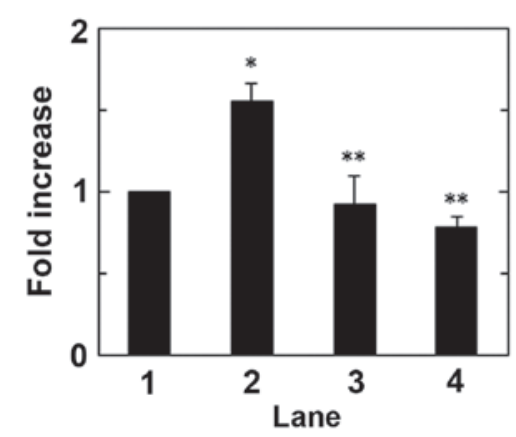

Figure 2. Effect of AICAR on the collagen-induced phosphorylation of p44/p42 MAPK in human platelets. (A) Following treatment with various doses of AICAR for $15 \mathrm{~min}$ and stimulation by $1.0 \mu \mathrm{g} / \mathrm{ml}$ collagen or vehicle for $5 \mathrm{~min}$, the lysates of platelets were subjected to western blot analysis using antibodies against p-p44/p42 MAPK, p44/p42 MAPK or GAPDH. (B) Quantitative analysis of the levels of collagen-induced phosphorylation of p44/p42 MAPK, obtained from laser densitometric analysis of quadruplicate independent determinations. Data are presented as the mean \pm standard error of the mean of quadruplicate determinations. ${ }^{*} \mathrm{P}<0.05$, vs. the control; ${ }^{* *} \mathrm{P}<0.05$, vs. the collagen alone. AICAR, 5-aminoimidazole-4-carboxamide -1- $\beta$-d-ribofuranosyl 5'-monophosphate; MAPK, mitogen-associated protein kinase; p-, phosphorylated.

Therefore, in the present study the effects of AICAR on the secretion of PDGF-AB and the release of $\mathrm{SCD} 40 \mathrm{~L}$ induced by collagen were examined. AICAR administration significantly suppressed PDGF-AB secretion in a dose-dependent manner up to $1.0 \mu \mathrm{M}$, as compared with the control $(\mathrm{P}<0.05$; Fig. 4). The maximum inhibitory effect induced by $1.0 \mu \mathrm{M}$ AICAR on the collagen-induced PDGF-AB secretion was $\sim 60 \%$ a reduction in the collagen-effect. Furthermore, treatment with $1.0 \mu \mathrm{M}$ AICAR significantly reduced the collagen-stimulated release of SCD40L from human platelets, as compared with the control $(\mathrm{P}<0.05)$; however treatment with 1.0 $\mu \mathrm{M}$ AICAR did not induce a significant reduction. The maximum suppressive effect induced by $1.0 \mu \mathrm{M}$ AICAR was a $\sim 40 \%$ reduction in the collagen-effect (Fig. 5). These findings indicate that AMPK activates AICAR, simultaneously inhibiting PDGF-AB secretion and $\mathrm{SCD} 40 \mathrm{~L}$ release from human platelets, stimulated by collagen.

\section{Discussion}

The present study investigated the effect of AICAR, which is an AMPK activator (23), on the collagen-induced activation of human platelets. Using an aggregometer with a laser light scattering system, which can simultaneously detect both the light-transmittance and the size of platelet aggregates, the effect of AICAR on the aggregation of human platelets was assessed. The results demonstrated that AICAR administration inhibited collagen-induced platelet aggregation and decreased the collagen-induced formation of large aggregates (50-70 $\mu \mathrm{m})$ and increased the formation of small aggregates $(9-25 \mu \mathrm{m})$. Alterations in aggregate size are recognized to be more sensitive than the change of transmittance in platelet reactivity (26). Therefore, these findings suggested that AMPK-activating AICAR may inhibit collagen-stimulated human platelet aggregation.

We have previously reported that the collagen-induced phosphorylation of HSP27 via p44/p42 MAP kinase is directly proportional to the secretion of PDGF-AB and the release of sCD40L from human platelets (17). On the basis of these findings, the present study examined the effects of AICAR on the phosphorylation of HSP27 and p44/p42 MAP kinase induced by collagen in human platelets. The results of the present study demonstrated that AICAR markedly suppressed the expression levels of phosphorylated p44/p42 MAP kinase, suggesting that AMPK-activating AICAR negatively regulates the collagen-stimulated activation of p44/p42 MAP kinase in human platelets. Furthermore, the present study demonstrated that AICAR administration attenuated the phosphorylation of HSP27. These findings suggested that AMPK-activating AICAR may limit the phosphorylation of HSP27 via the suppression of p44/p42 MAP kinase activity in collagen-stimulated human platelets. The results of the present study also showed that the collagen-induced granule secretion of PDGF-AB was significantly reduced by pretreatment with AICAR. In addition, the collagen-stimulated SCD40L release was also suppressed by AICAR. Therefore, these findings indicated that AMPK-activating AICAR may simultaneously inhibit PDGF-AB secretion and SCD40L release from human platelets stimulated by collagen. It is most likely that AICAR negatively regulates the collagen-stimulated secretion of PDGF-AB and the release of SCD40L from human platelets through the inhibition of HSP27 phosphorylation via p44/p42 MAP kinase and platelet aggregation.

It is well recognized that PDGF-AB, which is released from $\alpha$-granules in activated-platelets, is a potent growth factor that promotes atherosclerosis (27). Furthermore, it has been demonstrated that $\mathrm{SCD} 40 \mathrm{~L}$, which is released from activated platelets $(28,29)$, induces inflammatory responses in vascular endothelial cells and neutrophils via CD40 (30), resulting in the progression of atherosclerosis (27). It has been reported that the elevation of plasma $\mathrm{SCD} 40 \mathrm{~L}$ is a risk factor for cardiovascular events in patients with unstable coronary artery disease (31). Therefore, a simultaneous reduction in PDGF-AB secretion, $\mathrm{SCD} 40 \mathrm{~L}$ release and platelet aggregation may be beneficial to the prohibition of atherosclerosis-related diseases, including acute coronary syndrome and stroke. The results of the present study may provide a novel therapeutic strategy for the treatment of these diseases. Notably, it has previously been demonstrated metformin, which is an AMPK-activating substance that is widely used to treat DM, is capable of inducing a reduction in cardiovascular events and ischemic stroke characterized by atherosclerosis (32). It is well known that DM is a risk factor of cardiovascular and cerebrovascular ischemic diseases (33). Regarding the function of platelets in DM, we have previously reported that the phosphorylation levels of collagen-induced p38 MAP kinase 
A

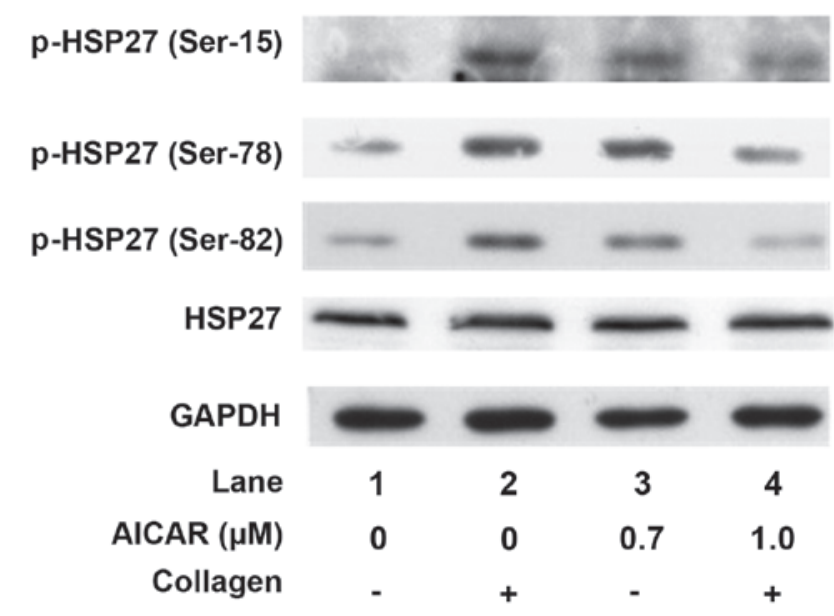

B
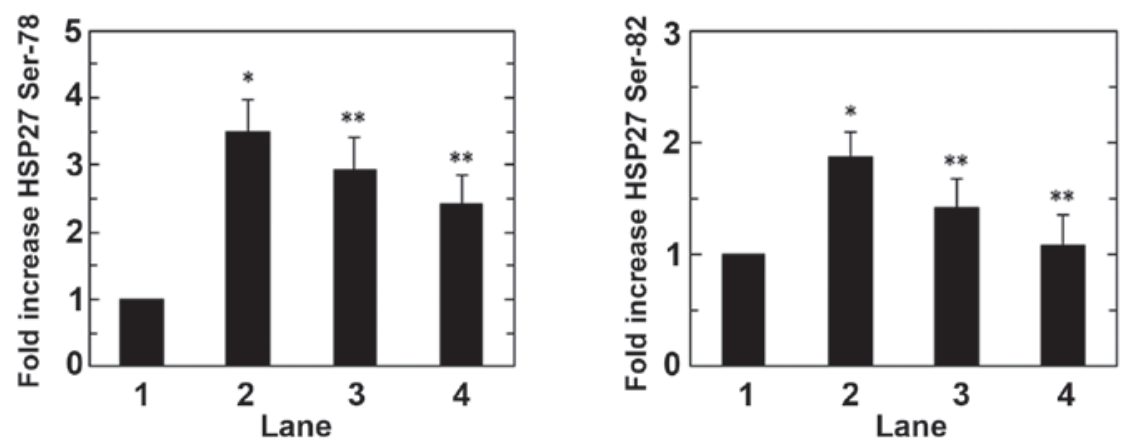

Figure 3. Effect of AICAR on the collagen-induced phosphorylation of HSP27 in human platelets. (A) Following treatment with various doses of AICAR for $15 \mathrm{~min}$ and stimulation by $1.0 \mu \mathrm{g} / \mathrm{ml}$ collagen or vehicle for $5 \mathrm{~min}$, the lysates of platelets were subjected to western blot analysis using antibodies against p-HSP27 (Ser-15, Ser-78 and Ser-82), HSP27 or GAPDH. (B) Quantitative analysis of the levels of collagen-induced phosphorylation of HSP27 (Ser-78 and Ser-82), obtained from laser densitometric analysis of quadruplicate independent determinations. Data are presented as the mean \pm standard error of the mean of quadruplicate determinations. ${ }^{*} \mathrm{P}<0.05$, vs. the control; ${ }^{* *} \mathrm{P}<0.05$, vs. the collagen alone. AICAR, 5 -aminoimidazole-4-carboxamide-1- $\beta$-d-ribofuranosyl 5'-monophosphate; p-, phosphorylated; HSP, heat shock protein.

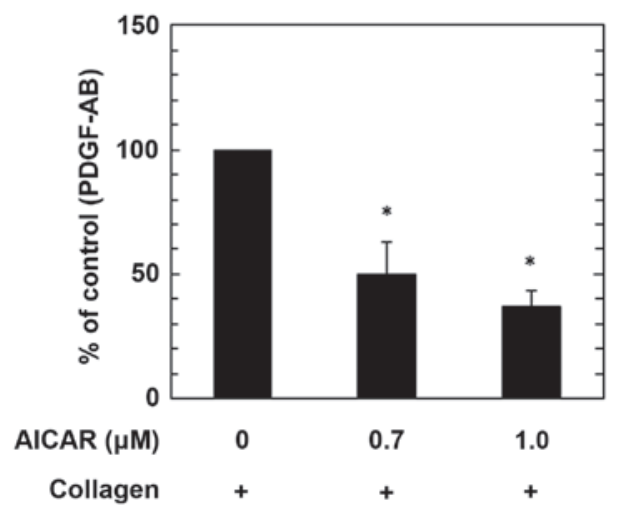

Figure 4. Effect of AICAR on collagen-induced PDGF-AB secretion from human platelets. Following treatment with various doses of AICAR at for $15 \mathrm{~min}$ and stimulation by $1.0 \mu \mathrm{g} / \mathrm{ml}$ collagen or vehicle for $5 \mathrm{~min}$, the mixture was centrifuged at $10,000 \mathrm{x}$ f for $2 \mathrm{~min}$ at $4^{\circ} \mathrm{C}$, and the supernatants were subjected to ELISA for PDGF-AB. Net increased levels of collagen alone were presented as $100 \%$. Data are presented as the mean \pm standard error of the mean of quadruplicate independent experiments. ${ }^{*} \mathrm{P}<0.05$, vs. collagen alone. AICAR, 5-aminoimidazole-4-carboxamide-1- $\beta$-d-ribofuranosyl 5 '-monophosphate; PDGAF, platelet-derived growth factor.

and $\mathrm{p} 44 / \mathrm{p} 42$ MAP kinase are correlated with the hyperaggregability of platelets derived from patients (34). Therefore, the ameliorating effect of an AMPK-activating agent on vascular events in DM may be due to the suppression of p44/p42 MAP

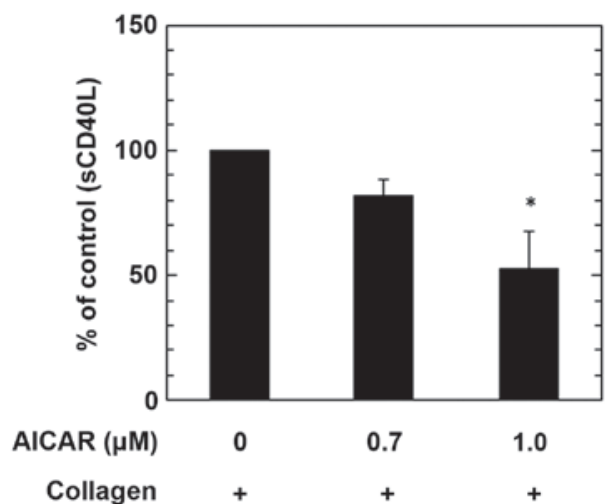

Figure 5. Effect of AICAR on collagen-induced SCD40L release from human platelets. Following treatment with various doses of AICAR for $15 \mathrm{~min}$ and stimulation by $1.0 \mu \mathrm{g} / \mathrm{ml}$ collagen or vehicle for $5 \mathrm{~min}$, the mixture was centrifuged at $10,000 \mathrm{xg}$ at $4^{\circ} \mathrm{C}$ for $2 \mathrm{~min}$, and the supernatants were subjected to ELISA for sCD40L. Net increased levels of collagen alone were presented as $100 \%$. Data are presented as the mean \pm standard error of the mean of quadruplicate independent experiments. ${ }^{*} \mathrm{P}<0.05$, vs. collagen alone. AICAR, 5-aminoimidazole-4-carboxamide-1- $\beta$-d-ribofuranosyl 5'-monophosphate; sCD40L, soluble cluster of differentiation 40 ligand.

kinase in human platelets; this suppression would result in the reduction of HSP27 phosphorylation associated with aggregation and the release of pro-inflammatory substances from platelets. Further investigation is required in order to clarify 
the molecular mechanisms underlying the effects of AMPK on human platelets.

In conclusion, the results of the present study suggested that AICAR-activated AMPK may reduce the collagen-induced secretion of PDGF-AB and release of SCD40L by inhibiting HSP27 phosphorylation via p44/p42 MAP kinase in human platelets.

\section{Acknowledgements}

The authors of the present study would like to thank Mrs. Yumiko Kurokawa for her skillful technical assistance. The present study was supported by grants from the Ministry of Education, Science, Sports and Culture of Japan (grant no. 23592249), and the Research Fund for Longevity Sciences (25-4) from National Center for Geriatrics and Gerontology in Japan (grant no. 26462335).

\section{References}

1. Davì G and Patrono C: Platelet activation and atherothrombosis. N Eng J Med 357: 2482-2494, 2007.

2. Nieswandt B and Watson SP: Platelet-collagen interaction: Is GPVI the central receptor? Blood 102: 449-461, 2003.

3. Moroi M and Jung SM: Platelet glycoprotein VI: Its structure and function. Thromb Res 114: 221-233, 2004.

4. Zheng YM, Liu C, Chen H, Locke D, Ryan JC and Kahn ML: Expression of the platelet receptor GPVI confers signaling via the $\mathrm{Fc}$ receptor gamma-chain in response to the snake venom convulxin but not to collagen. J Biol Chem 276: 12999-13006, 2001.

5. Berlanga O, Tulasne D, Bori T, Snell DC, Miura Y, Jung S, Moroi M, Frampton J and Watson SP: The Fc receptor gamma-chain is necessary and sufficient to initiate signalling through glycoprotein VI in transfected cells by the snake C-type lectin, convulxin. Eur J Biochem 269: 2951-2960, 2002.

6. Blake RA, Schieven GL and Watson SP: Collagen stimulates tyrosine phosphorylation of phospholipase C-gamma 2 but not phospholipase C-gamma 1 in human platelets. FEBS Lett 353: 212-216, 1994.

7. Yanaga F, Poole A, Asselin J, Blake R, Schieven GL, Clark EA, Law CL and Watson SP: Syk interacts with tyrosine-phosphorylated proteins in human platelets activated by collagen and cross-linking of the Fc gamma-IIA receptor. Biochem J 311: 471-478, 1995.

8. Shattil SJ and Newman PJ: Integrins: Dynamic scaffolds for adhesion and signaling in platelets. Blood 104: 1606-1615, 2004.

9. Hendrick JP and Hartl FU: Molecular chaperone functions of heat-shock proteins. Annu Rev Biochem 62: 349-384, 1993.

10. Kampinga HH, Hageman J, Vos MJ, Kubota H, Tanguay RM, Bruford EA, Cheetham ME, Chen B and Hightower LE: Guidelines for the nomenclature of the human heat shock proteins. Cell Stress Chaperones 14: 105-111, 2009.

11. Kato K, Ito $\mathrm{H}$ and Inaguma Y: Expression and phosphorylation of mammalian small heat shock proteins. Prog Mol Subcell Biol 28: 129-150, 2002.

12. Koteiche HA and McHaourab HS: Mechanism of chaperone function in small heat-shock proteins. Phosphorylation-induced activation of two-mode binding in alpha B-crystallin. J Biol Chem 278: 10361-10367, 2003.

13. Acunzo J, Katsogiannou M and Rocchi P: Small heat shock proteins HSP27 (HspB1), $\alpha \mathrm{B}$-crystallin (HspB5) and HSP22 (HspB8) as regulators of cell death. Int J Biochem Cell Biol 44 $1622-1631,2012$.

14. Merendino AM, Paul C, Vignola AM, Costa MA, Melis M, Chiappara G, Izzo V, Bousquet J and Arrigo AP: Heat shock protein-27 protects human bronchial epithelial cells against oxidative stress-mediated apoptosis: Possible implication in asthma. Cell Stress Chaperones 7: 269-280, 2002.
15. Rogalla T, Ehrnsperger M, Preville X, Kotlyarov A, Lutsch G, Ducasse C, Paul C, Wieshe M, Arrigo AP, Buchner J and Gaestel M: Regulation of Hsp27 oligomerization, chaperone function, and protective activity against oxidative stress/tumor necrosis factor alpha by phosphorylation. J Biol Chem 274: 18947-18956, 1999.

16. Saklatvala J, Rawlinson L, Waller RJ, Sarsfield S, Lee JC, Morton LF, Barnes MJ and Farndale RW: Role for p38 mitogen-activated protein kinase in platelet aggregation caused by collagen or a thromboxane analogue. J Biol Chem 271: 6586-6589, 1996.

17. Kato H, Adachi S, Doi T, Matsushima-Nishiwaki R, Minamitani C, Akamatsu S, Enomoto Y, Tokuda H, Otsuka T, Iwama T, et al: Mechanism of collagen-induced release of 5-HT, PDGF-AB and sCD40L from human platelets: Role of HSP27 phosphorylation via p44/p42 MAPK. Thromb Res 126: 39-43, 2010.

18. Hardie DG: AMP-activated protein kinase as a drug target. Annu Rev Pharmacol Toxicol 47: 185-210, 2007.

19. Steinberg GR and Kemp BE: AMPK in Health and Disease. Physiol Rev 89: 1025-1078, 2009.

20. Wong AK, Howie J, Petrie JR and Lang CC: AMP-activated protein kinase pathway: A potential therapeutic target in cardiometabolic disease. Clin Sci (Lond) 116: 607-620, 2009.

21. Zhang BB, Zhou G and Li C: AMPK: An emerging drug target for diabetes and the metabolic syndrome. Cell Metab 9: 407-416, 2009.

22. Liu Y, Oh SJ, Chang KH, Kim YG and Lee MY: Antiplatelet effect of AMP-activated protein kinase activator and its potentiation by the phosphodiesterase inhibitor dipyridamole. Biochem Pharmacol 86: 914-925, 2013.

23. Cool B, Zinker B, Chiou W, Kifle L, Cao N, Perham M, Dickinson R, Adler A, Gagne G, Iyengar R, et al: Identification and characterization of a small molecule AMPK activator that treats key components of type 2 diabetes and the metabolic syndrome. Cell Metab 3: 403-416, 2006.

24. Kato K, Ito H, Hasegawa K, Inaguma Y, Kozawa O and Asano T: Modulation of the stress-induced synthesis of hsp27 and alpha B-crystallin by cyclic AMP in C6 rat glioma cells. J Neurochem 66: 946-950, 1996

25. Laemmli UK: Cleavage of structural proteins during the assembly of the head of bacteriophage T4. Nature 227: 680-685, 1970.

26. Fusegawa Y and Handa S: Platelet aggregation induced by ADP or epinephrine is enhanced in habitual smokers. Thromb Res 97: 287-295, 2000.

27. Heldin $\mathrm{CH}$ and Westermark B: Mechanism of action and in vivo role of platelet-derived growth factor. Physiol Rev 79: 1283-1316, 1999.

28. Hermann A, Rauch BH, Braun M, Schrör K and Weber AA: Platelet CD40 ligand (CD40L)-subcellular localization, regulation of expression, and inhibition by clopidogrel. Platelets 12: 74-82, 2001.

29. André P, Nannizi-Alaimo L, Prasad SK and Phillips DR: Platelet-derived CD40L: The switch-hitting player of cardiovascular disease. Circulation 106: 896-899, 2002.

30. Henn V, Slupsky JR, Gräfe M, Anagnostopoulos I, Förster R, Müller-Berghaus G and Kroczek RA: CD40 ligand on activated platelets triggers an inflammatory reaction of endothelial cells. Nature 391: 591-594, 1998.

31. Heeschen C, Dimmeler S, Hamm CW, van den Vrand MJ, Boersma E, Zeiher AM and Simoons ML; CAPTURE study Investigators: Soluble CD40 ligand in acute coronary syndrome. N Eng J Med 348: 1104-1111, 2003.

32. Chang CH, Chang YC, Lin JW, Chen ST, Chuang LM and Lai MS: Cardiovascular risk associated with acarbose versus metformin as the first-line treatment in patients with type 2 diabetes: A Nationwide Cohort Study. J Clin Endocrinol Metab 100: 1121-1129, 2015.

33. Emerging Risk Factors Collaboration; Sarwar N, Gao P, Seshasai SR, Gobin R, Kaptoge S, Di Angelantonio E, Ingelsson E, Lawlor DA Selvin E, et al: Diabetes mellitus, fasting blood glucose concentration, and risk of vascular disease: A collaborative meta-analysis of 102 prospective studies. Lancet 375: 2215-2222, 2010.

34. Hanai Y, Adachi S, Yasuda I, Takai S, Matsushima-Nishiwaki R, Kato H, Enomoto Y, Akamatsu S, Sakakibara S, Ogura S, et al: Collagen-induced p38 MAP kinase activation is a biomarker of platelet hyper-aggregation in patients with diabetes mellitus. Life Sci 85: 386-394, 2009. 